\title{
Marchés, produits et innovation
}

Markets, products and innovation

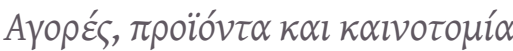

\section{Christina Agriantoni}

\section{CpenEdition}

\section{Journals}

Édition électronique

URL : https://journals.openedition.org/ceb/10481

DOI : 10.4000/ceb.10481

ISSN : 2261-4184

Éditeur

INALCO

Édition imprimée

ISBN : 978-2-85831-294-8

ISSN : 0290-7402

Référence électronique

Christina Agriantoni, « Marchés, produits et innovation », Cahiers balkaniques [En ligne], 45 | 2018, mis en ligne le 14 décembre 2018, consulté le 10 juillet 2021. URL : http://journals.openedition.org/ceb/ 10481 ; DOI : https://doi.org/10.4000/ceb.10481

Ce document a été généré automatiquement le 10 juillet 2021.

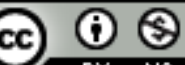

Cahiers balkaniques est mis à disposition selon les termes de la Licence Creative Commons Attribution - Pas d'Utilisation Commerciale 4.0 International. 


\title{
Marchés, produits et innovation
}

\author{
Markets, products and innovation

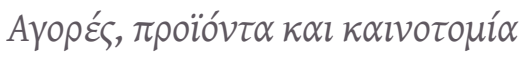

Christina Agriantoni

1 Depuis longtemps déjà, la grande entreprise américaine n'est plus le modèle universel d'efficacité industrielle. L'émergence des nouveaux pays industriels a ouvert les horizons de la pensée historique, et l'on a pu découvrir les "variétés du capitalisme », les multiples voies possibles de l'industrialisation, et mettre un terme à la disqualification d'une série de branches autres que celles supposées être à la pointe du progrès technologique et organisationnel. Philip Scranton a montré, il y a presque vingt ans, que, même aux États-Unis, ce type d'entreprise managériale, taylorienne et aux produits standardisés n'a pas été la règle ${ }^{1}$. En même temps, Gérard Chastagnaret évoquait la nécessité de reconsidérer l'industrialisation en Méditerranée ${ }^{2}$.

\section{Les structures de l'industrie grecque de la fin du XIX $X^{\mathrm{e}}$ siècle à 1970}

2 Pour parler du cas de la Grèce dans un cadre méditerranéen et comparatif, il me semble propice de centrer mon exposé sur une condition qui lui est plus ou moins particulière, à savoir les dimensions restreintes du marché national. La Grèce, on le sait, est un petit pays, qui était encore plus petit au xixe siècle - moins de la moitié du territoire actuel. Environ un million et demi d'âmes en 1870, deux millions, après l'annexion de la Thessalie en 1881, 5,5 millions en 1920 après l'annexion de la Macédoine, 6,2 millions en 1928, après l'installation des réfugiés d'Asie Mineure. C'est, en plus, une économie où domine le secteur agricole au moins jusqu'en 1940, la population rurale (agglomérations de moins de 2000 habitants) représentant encore 52,4\% du total en 1940.

3 Sans doute, la taille du marché ne peut-elle être un critère unique pour des comparaisons pertinentes. Il suffit d'essayer de comparer l'industrialisation de la Grèce avec celle d'autres petits pays (du point de vue du marché) de l'Europe, comme les pays 
scandinaves ou les pays balkaniques, pour se rendre compte du fait que les convergences ou les divergences s'établissent sur la base d'autres facteurs, comme l'histoire politique, la culture technique ou les ressources naturelles. Et pourtant, la taille du marché est un élément capital pour comprendre les comportements et les stratégies des entrepreneurs. On oublie souvent, dans les études sur la grande entreprise industrielle née aux États-Unis vers la fin du xIX siècle, que ce type, ce format, comme dirait Scranton, d'unité de production qu'a décrit et glorifié Alfred Chandler, n'aurait pas pu émerger sans la perspective d'un grand marché.

4 Les choix, les comportements des industriels en Grèce face au problème des débouchés émergent à la croisée de l'étroitesse du marché local, de la technologie et de la nature des produits. Avant de les examiner, il faut rappeler, en bref, quelques traits essentiels de l'industrialisation de la Grèce - en commençant par son timing. Les premières cheminées d'usines se dressent au cours des années 1860-1870 : textile (coton et soie), minoterie, savon, cuir, alimentaire, constructions mécaniques, secteur minier ${ }^{3}$. Ce démarrage coïncide avec celui de plusieurs pays de la périphérie européenne, mais aussi du Moyen-Orient et du Japon. La seconde vague, beaucoup plus importante, diffuse et multiforme, démarre vers la fin du XIX ${ }^{e}$ siècle et se combine avec la crise agricole et l'exode rural. Chimie, électricité, ciment sont les nouveaux acquis de cette période, ainsi qu'une vague de fusions et l'émergence des premières grandes entreprises. Après les anomalies de la décennie de guerres (balkaniques, mondiale, gréco-turque), l'élan donné par l'agrandissement du pays, puis par l'installation des réfugiés (élargissement du marché donc), prolonge cette vague tout au long de l'entre-deux-guerres. Peu de nouveautés dans l'ensemble au niveau des branches: automobile (carrosserie), bauxite, tabac, phonographie, cinéma ${ }^{4}$. Après la Seconde Guerre mondiale, les années 1955-1975 représentent l'âge d'or de l'industrie grecque, en particulier avec de gros investissements avec du capital étranger - l'usine de Pechiney est le fleuron de la période - et les secteurs remarquables des constructions navales, de la métallurgie de l'acier, de l'automobile (assemblage), et de l'industrie pharmaceutique ${ }^{5}$. Par la suite, les grands bouleversements, connus, de l'économie au niveau mondial ont affecté l'industrie en Grèce comme en Europe, et je ne me hasarderai pas dans cette période récente dont l'aboutissement est plutôt douloureux pour le pays. Il serait cependant utile pour mon propos de signaler que les survivants, parmi les entreprises d'une certaine taille tout au moins, sont en grande majorité ceux qui ont réussi à sortir des frontières du pays, c'est-à-dire à gagner des parts de marché à l'étranger.

5 Voilà pour la temporalité de l'industrialisation. Quant à son caractère, elle se rapproche de ce type d'industrialisation labour intensive qui, d'après des études récentes, a été le modèle dominant de l'industrialisation des pays asiatiques, un modèle opposé à l'industrialisation capital intensive britannique ou américaine ${ }^{6}$. En effet, à l'exception d'une bonne partie du xix siècle, lorsque le développement dynamique du secteur agricole exportateur absorbait toutes les disponibilités, le facteur travail a été plutôt abondant en Grèce, à cause du déclin du secteur agricole à partir de la fin du XIX ${ }^{e}$ siècle, puis, pendant l'entre-deux-guerres, grâce à l'arrivée des réfugiés.

6 Ensuite, il faut rappeler que malgré la dispersion, en milieu rural, d'une foule de petites unités de traitement des produits agricoles, l'industrialisation en Grèce a été une affaire de villes, dont certaines sont même devenues de vraies villes industrielles se rapprochant du modèle des clusters où l'on rencontre des interdépendances entre 
firmes, des économies externes engendrées par le voisinage, voire des formes de coopération comme les transferts de commandes ou les sous-traitances, l'usage partagé de machines spéciales, la cession temporaire d'espaces disponibles au voisin, etc. Si les petites entreprises familiales représentent la grande majorité, les grandes entreprises et les grandes dynasties familiales ne manquent pas. Et enfin, si les secteurs du textile et de l'agroalimentaire ont été largement dominants jusqu'à la Seconde Guerre mondiale, il y a eu en même temps une impressionnante variété d'industries lancées dans le pays, ne serait-ce qu'à petite échelle. En dehors de la rapidité de la circulation de l'information technologique ${ }^{7}$, il faudrait attribuer ce phénomène à une idéologie bien ancrée parmi les élites politiques et sociales qui étaient favorables à la modernisation et à l'européanisation, mais aussi, probablement, à la promptitude du monde entrepreneurial à chercher de nouvelles opportunités.

\section{Les entreprises face au problème des débouchés}

\section{La diversité des productions}

7 En effet, le premier élément du comportement des entreprises face au problème des débouchés, c'est la diversité de leurs productions. On sait maintenant, grâce à plusieurs études, qu'il s'agit d'une stratégie (differentiation strategy) de survie ou de croissance, qui est l'opposé, dans tous les cas, de la stratégie de la réduction des coûts à travers la standardisation et l'augmentation de la productivité. Les constructions mécaniques représentent l'exemple le plus typique de cette stratégie de diversification que l'on rencontre d'ailleurs dans d'autres pays méditerranéens ${ }^{8}$; mais ils ne sont pas les seuls : Jonathan Zeitlin a montré les limites de l'américanisation de l'industrie mécanique européenne, en étudiant le cas des constructeurs britanniques qui ont refusé, après la Seconde Guerre mondiale, d'abandonner leur capacité à produire des machines et outils très divers contre une standardisation plus poussée, à coûts réduits, comme le conseillaient les experts américains ${ }^{9}$. Sans doute, le problème des Britanniques n'était-il pas, comme dans notre cas, l'insuffisance des débouchés, mais la volonté de pouvoir répondre à la demande très diversifiée d'une clientèle mondiale.

Les constructeurs grecs ont pourtant essayé, dès leurs débuts, de fabriquer des articles en série, signe qu'ils en comprenaient les avantages. Il apparaît que les moulins à huile représentaient une importante clientèle potentielle qu'ils ont essayé de gagner avec des pressoirs en fer. On rencontre même deux efforts successifs d'invention technologique dans ce domaine $(1888,1905)$, par deux constructeurs du Pirée, concernant une machine à extraire les noyaux des olives qui a été présentée même en Italie et brevetée en France et en Angleterre ${ }^{10}$. À la même époque, une grosse fonderie du Pirée a essayé de standardiser la production de chaudières. Ces démarches n'ont pas été concluantes. La concurrence s'est vite développée pour les pressoirs à huile, les machines inventées étaient défectueuses et, quant aux chaudières, la concurrence des Babcock \& Wilcox, massivement importées alors par les industries, était très forte.

9 L'industrie mécanique a donc résolument adopté la stratégie de la diversité des productions et de la flexibilité. Concentrés surtout au Pirée, mais aussi à Ermoúpolis, Volos, plus tard à Thessalonique, les fonderies et les ateliers de mécanique fabriquaient toutes sortes d'outils et d'éléments d'équipement industriel sur commande, des répliques de machines importées, des articles pour le bâtiment (balustrades en fer...); 
ils offraient des services de réparation et de maintien aux industries de la place, et vers la fin de l'entre-deux-guerres, certains ont même entamé la production de petits moteurs à pétrole. Les plus grandes entreprises du secteur ont accaparé les trois débouchés les plus importants, en parfaite harmonie avec ce profil de travail sur commande: constructions et réparations navales, pour les entreprises du Pirée et d'Ermoúpolis, grandes constructions métalliques des travaux publics, pour les constructeurs d'Athènes et du Pirée, instruments, petites machines et roues hydrauliques destinées la clientèle rurale, pour ceux de Volos. La diversification a été d'ailleurs la règle dans l'ensemble du secteur des métaux avant la Seconde Guerre mondiale; de grands noms des fabricants de l'électroménager de l'après-guerre, comme Pitsos ou Izola, ou même de la métallurgie de nos jours comme Mytilinaios, débutèrent alors avec des ferblanteries aux produits et services très divers.

Les industries de traitement des produits agricoles d'exportation n'avaient pas en réalité de problème de débouchés. Dans ce cas, leur trait principal était le lien très étroit entre le commerce et l'industrie, et la possibilité de repli de l'un à l'autre. Car, en fait, l'apparition même de ces industries a été le résultat des pressions exercées par les marchés extérieurs sur les prix. Ainsi l'égrenage mécanique du coton a débuté avec la flambée des prix du coton et l'extension de la culture en Grèce au cours des années 1860 ; le triage mécanique et l'emballage du raisin sec sont apparus à leur tour au moment de la baisse des prix du raisin sec au cours des années 1890 ; les premières machines à cigarettes ont été introduites au temps de la montée des prix du tabac au début $\mathrm{du} \mathrm{xx}^{\mathrm{e}}$ siècle tandis qu'à l'inverse, les nouvelles méthodes d'emballage du tabac exporté en feuilles ont été introduites dans les grands entrepôts du Nord du pays pendant la dépression de l'entre-deux-guerres. Et évidemment, pour l'ensemble du secteur minier, l'exploitation des gisements a suivi les fluctuations du mouvement international des prix des matières premières concernées ${ }^{11}$.

11 Mais on retrouvait la diversité lorsqu'il fallait s'adresser au marché intérieur.

\section{Diversification par l'introduction de nouveaux produits}

Diversification signifiait souvent introduction de nouveaux produits: la Société des Vins et Spiritueux, fondée au début $\mathrm{du} \mathrm{xx}^{\mathrm{e}}$ siècle pour exploiter les tonnes de raisins secs invendus qui s'entassaient dans les dépôts publics au titre de " parakratima » (une retenue à la source pour alléger le marché saturé et soutenir les prix) a lancé sur le marché l'alcool de raisin sec pour éclairage et pour des moteurs spécialement adaptés, en dehors de l'alcool pour boissons, des gâteaux et marmelades de raisin, et des vins à marque commerciale (Votrys). En mixage avec de l'essence de térébenthine, cet alcool a alimenté les moteurs des voitures au cours de la Grande Guerre, en raison du manque d'essence.

La diversification par le lancement de nouveaux produits a été la principale condition de croissance de l'industrie huilière. Les méthodes traditionnelles de production de l'huile d'olive par des milliers de petits moulins disséminés sur la plus grande partie du territoire qui délivraient à chaque producteur ses propres tonneaux d'huile tirée de sa récolte personnelle (système qui fonctionne encore de nos jours, d'ailleurs), ne laissaient guère de marge d'action aux entrepreneurs intéressés par des secteurs autres que le négoce de l'huile. Mais, déjà à ce niveau, on décèle une innovation importante, lorsque deux négociants athéniens qui achetaient leur huile au sud du Péloponnèse ont 
introduit, les premiers vers 1904, l'emballage en boîtes métalliques avec étiquette et la marque (le brand name) Minerva. Il n'est pas sans intérêt de noter que "Minerva " exportait aux États-Unis, c'est-à-dire aux Grecs des États-Unis, à savoir le marché de la diaspora hellénique, point sur lequel il faudra revenir. À noter aussi que "Minerva ", aujourd'hui une des firmes d'huile bien connues en Grèce, est longtemps demeurée une firme commerciale et n'a fondé son installation de raffinage qu'en $1957^{12}$. En fait, l'industrie huilière, qui a commencé effectivement par le raffinage au cours des années 1920, s'est développée en contournant l'huile d'olive pour se tourner vers les graines oléagineuses (de lin, coton, arachides, palme, sésame) et proposer une gamme constamment élargie de nouveaux produits, des substituts moins chers de l'huile d'olive ou du beurre; ce furent les diverses huiles de graines, les graisses végétales (c'est ELAIS qui a lancé le premier brand name, la Fytini, en 1932), puis, après la guerre, les margarines (le VITAM, encore par ELAIS la plus grande firme du secteur, absorbée par Unilever en 1976) ${ }^{13}$.

14 Pour les industries des biens intermédiaires, industries le plus souvent capital intensives, le problème des débouchés se présentait sous la forme de l'offre précédant la demande, ce qui est souvent considéré comme un des changements importants du système productif, survenu au temps de la seconde révolution industrielle. Les industries du ciment, un bon exemple de ce cas de figure, introduites en Grèce au cours de la première décennie $d u x^{e}$ siècle avec une capacité qui excédait la demande (car la technologie de la production imposait un certain seuil pour que l'investissement soit rentable), ont essayé de faire face au problème en s'étendant vers l'aval, pour créer leur propre demande; presque dès leur début, des industriels du secteur ont fondé des entreprises de construction de bâtiment et de travaux publics. On peut trouver des exemples analogues dans la tannerie où certains industriels ont créé des entreprises de chaussures, ainsi que dans le cas de la société Réfrigérateurs du Pirée qui a créé une entreprise d'importation, traitement et vente de morues, en collaboration avec la plus grande industrie de bière (Fix), manifestement parce que tous les deux avaient une surcapacité en installations frigorifiques. L'autre solution, pour ces industries capital intensive, était la promotion systématique du produit et la monopolisation du marché. Ce fut la solution adoptée par la plus grande entreprise industrielle de la Grèce d'avant la Seconde Guerre mondiale, la Société des Engrais et Produits chimiques (1909), qui a mené toute une campagne pour promouvoir l'usage de ses engrais, avec publications spéciales, création de champs-modèles où les engrais étaient testés, et développement d'une politique de collaboration sous contrat avec les agriculteurs. S'étant assuré le monopole dans le domaine des engrais, la société a vu ses ventes multipliées par cinq entre 1932 et 1938. Par ailleurs, les cimenteries aussi ont essayé de monopoliser le marché, et des cinq entreprises des années 1920, il en est resté deux après la crise.

\section{Une industrie de biens de consommation}

Mais ce ne sont là que quelques cas plutôt exceptionnels. L'industrie grecque était majoritairement une industrie de biens de consommation, de produits finis. L'ancrage à la consommation assurait la capacité de sonder directement les tendances de la clientèle, les changements des goûts, et de s'adapter aux fluctuations d'un marché restreint et instable qui incitait donc à la flexibilité et la diversité des produits. Si l'on associe tout cela à la disponibilité du facteur travail, on comprend mieux pourquoi, dans le cas de chaînes productives de produits complexes comme l'automobile, les 
fabricants grecs de l'entre-deux-guerres se sont placés à la dernière étape de la chaîne, celle qui était encore plutôt labour intensive, pour fabriquer des carrosseries de toute sorte, en fonction des commandes, sur des châssis et des moteurs importés. Si après la Seconde Guerre mondiale quelques entreprises du secteur ont acquis une certaine importance en produisant des autobus et des poids lourds en collaboration avec les constructeurs européens des moteurs, elles ne se sont jamais spécialisées en un seul type de voiture. Ce n'est que dans les années 1970 que la première véritable chaîne de montage de voitures Nissan a été installée en Grèce (mais elle n'a pas survécu à la crise des années 1990).

\section{Conclusion}

Pour conclure, deux mots sur ce qui aurait pu être une alternative permettant de se débarrasser des contraintes d'un marché restreint: les exportations. Les industries grecques ne se sont pas trompées sur leur importance, lorsqu'elles ont essayé d'exporter dès leurs premiers pas au XIX ${ }^{e}$ siècle, et certaines, comme les filatures de coton ou les tanneries, y ont même réussi ; ce fut le cas également lorsque, à la fin du même siècle, au temps de la grande crise du raisin sec, elles ont été des dizaines à se bousculer dans la production et l'exportation de vins, du « cognac » grec ou de l'alcool de vin. Or, elles n'ont jamais réussi à gagner durablement des parts de marché à l'étranger. Les raisons en sont diverses, mais pour faire simple sur une question assez compliquée, on peut dire que, si l'on excepte l'affirmation finalement assez banale que, de toute façon, il serait très difficile pour l'industrie d'un pays late comer de concurrencer les champions de l'industrialisation, les raisons de cet échec ont été essentiellement politiques.

- Raisons de politique extérieure d'abord - ou de relations internationales : les exportations industrielles de la Grèce se sont principalement dirigées vers les pays voisins des Balkans, vers la Turquie et le Proche-Orient ou l'Égypte, à une époque où le coût des transports maritimes permettait encore de profiter de la proximité. Mais à partir de la fin du XIX $\mathrm{X}^{\mathrm{e}}$ siècle, cet avantage a été annulé par le développement des transports, et surtout, le déclin des communautés de la diaspora hellénique dans ces pays, qui étaient un point d'appui important des exportations, ainsi que le climat conflictuel - voire guerrier - qui s'installa dans la région au temps de la montée des nationalismes, ont perturbé les échanges et fait monter des barrières insurmontables aux exportations grecques. Seule la nouvelle diaspora hellénique aux États-Unis a offert un débouché durable, notamment aux industries agroalimentaires comme «Minerva», dont nous avons parlé, «Metaxás» (cognac) et d'autres.

- Politique interne ensuite : il s'agit tout simplement du fait que la classe politique grecque n'a jamais cru en la possibilité du développement industriel du pays avant la Seconde Guerre mondiale (mais en réalité, on trouve encore aujourd'hui les traces de cette mentalité). Il n'y a pas d'économie sans politique, même lorsque celle-ci brille par son absence. 


\section{BIBLIOGRAPHIE}

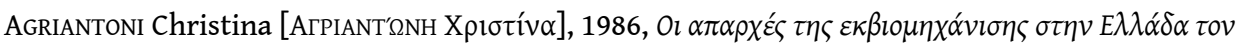

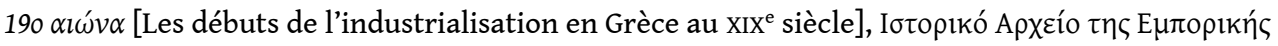

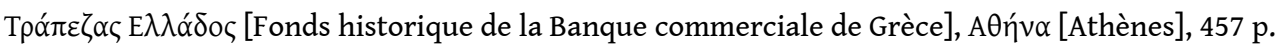

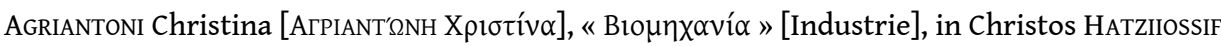

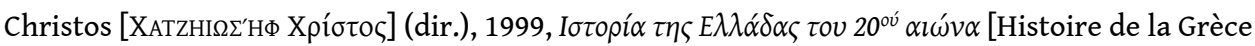

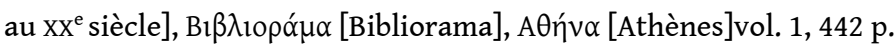

AUSTIN Gareth \& SUGIHARA Kaoru (eds), 2013, Labour-intensive industrialization in global history, Routledge, London, 314 p.

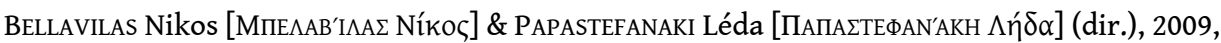

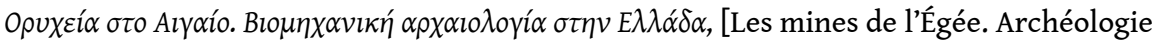

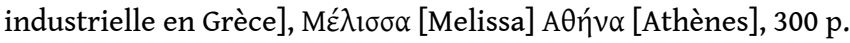

CHATzis Konstantinos, 2004, «La modernisation technique de la Grèce, de l'indépendance aux années de l'entre-deux-guerres : faits et problèmes d'interprétation », Études Balkaniques 3, p. 3-23.

ChASTAGnARet Gérard, 1997, «L'industrie en Méditerranée : une histoire en construction », Méditerranée, V : 87/3-4, p. 5-12.

DAUMALIN Xavier \& RAVEuX Olivier, 2001, « Marseille (1831-1865) ; une révolution industrielle entre Europe du Nord et Méditerranée », Annales HSS, 56/1, p. 153-176.

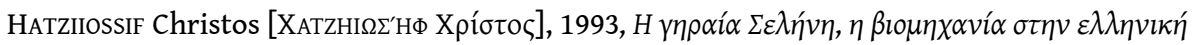

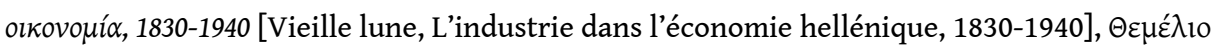
[Themelio], AӨńva [Athènes], $440 \mathrm{p}$.

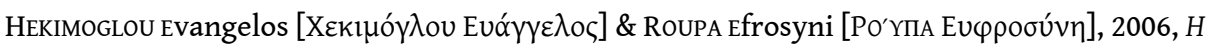

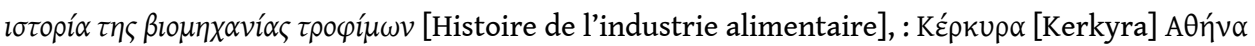
[Athènes], $332 \mathrm{p}$.

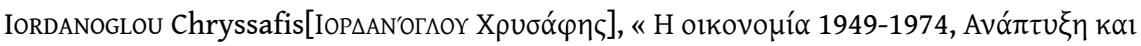

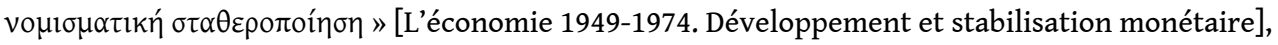

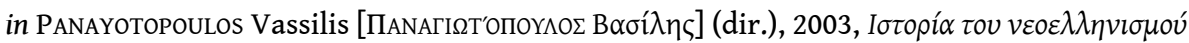

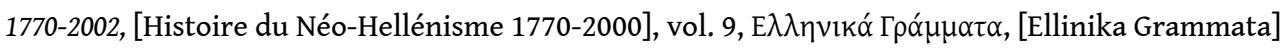

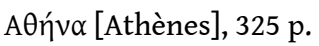

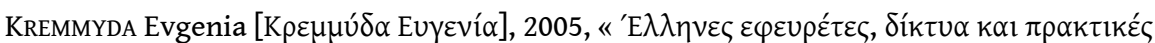

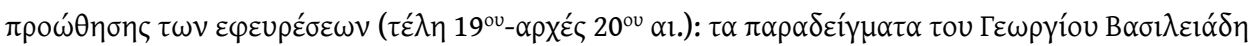

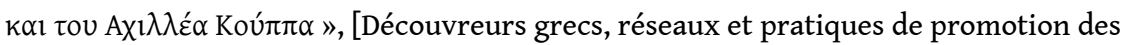
découvertes - fin $\mathrm{XIX}^{\mathrm{e}}$-début $\mathrm{XX}^{\mathrm{e}}$ siècle : les exemples de Georgios Vasiliadis et de

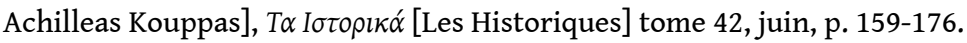

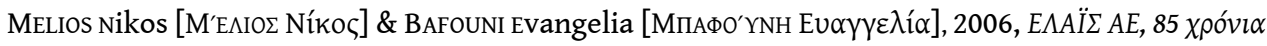

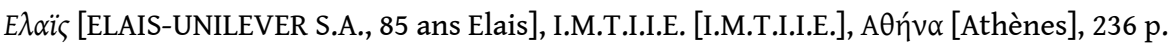

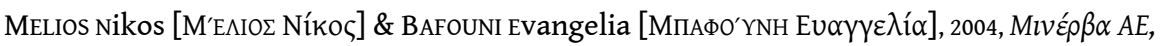
$100 \chi \rho o ́ v ı \alpha$ Mivé $\beta \alpha \alpha$ [Minerva S.A., 100 ans Minerva], I.M.T.I.I.E. [I.M.T.I.I.E.], A $\theta \eta ́ v \alpha$ [Athènes], $180 \mathrm{p}$. 
SCRANTON Philip, 1997, Endless Novelty: Specialty Production and American Industrialization 1865-1925, Princeton University Press, Princeton, 416 p.

ZEITLIN Jonathan, 1995, “Americanization and its limits: Theory and practice in the reconstruction of British engineering industries", Business and Economic History Review on line, Fall, pp. 277-286; version française : «L'industrie britannique des constructions mécaniques s'est-elle américanisée ? ", Histoire, Économie, Société, vol. 20/4 (2001), p. 546-576.

\section{NOTES}

1. SCRANTON, 1997.

2. Chastagnaret, 1997, p. 5-12.

3. AGRIANTONI, 1986.

4. HATZIIOSSIF, 1993 ; AGRIANTONI, 1999, vol. 1, p. 173-221.

5. IORDANOGLOU, 2003, vol. 9, p. 59-86.

6. Par ex. Austin \& Sugihara (eds), 2013.

7. Voir à ce sujet, CHATzIs, 2004, p. 3-23.

8. Daumalin \& Raveux, 2001, p. 153-176.

9. ZeITLIN, 1995, p. 277-286.

10. KREMMYDA, 2005, tome 42, p. 159-176.

11. Bellavilas \& Papastefanaki (dir.), 2009.

12. HeKIMOgLOU \& ROUPA, 2006 ; MELIOS \& BAFOUNI, 2004.

13. MeLios \& BAFOUNI, 2006.

\section{RÉSUMÉS}

Cette communication étudie le rapport entre marchés, produits et innovation en Grèce.

Jusqu'à une période récente, les industries grecques dépendaient presque entièrement du marché intérieur. C'est la raison principale pour laquelle elles avaient peu d'opportunités de croissance. Dans ce contexte, l'innovation portait presque exclusivement sur le produit, le lancement d'articles nouveaux et de services, dans la mesure où le marché intérieur pouvait les absorber.

Cette étude décrit en premier lieu la structure de l'industrie grecque de la fin du XIX ${ }^{\mathrm{e}}$ siècle jusqu'en 1970, examine ensuite les initiatives d'introduction de nouveaux produits sur le marché intérieur, en ce qui concerne la technologie importée et le marketing des nouveaux produits, discute enfin le problème des exportations industrielles grecques.

This paper studies the relationship between markets, products and innovation in Greece. Until recent period, the Greek industries depended almost completely on the internal market. It is the main reason for which the Greek companies had few growth opportunities. In this context, 
the innovation concerned almost exclusively the product, the launch of new articles and services, as far as the internal market could absorb them.

This study describes first the structure of the Greek industry from the end of the $19^{\text {th }}$ century until 1970, examine then the initiatives of introduction of new products in the internal market, as regards the imported appropriate technology and the marketing of the new products; it discusses finally the problem of the Greek industrial exports.

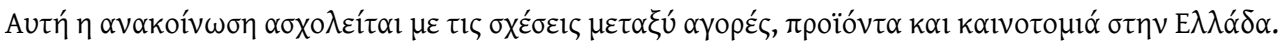

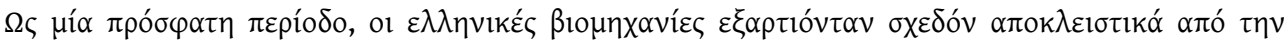

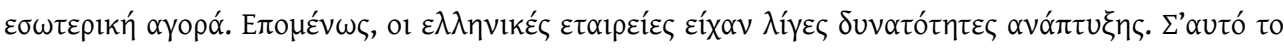

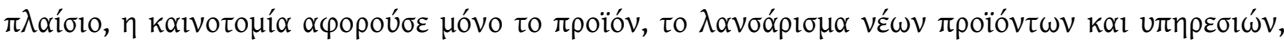

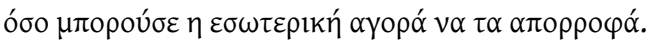

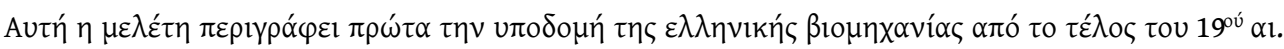

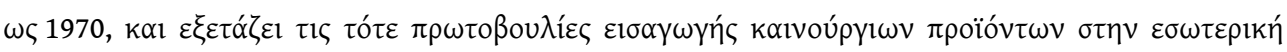

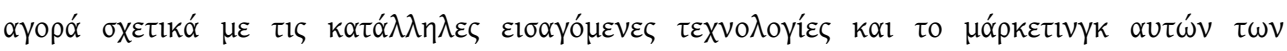

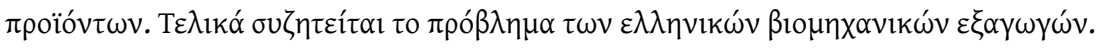

\section{INDEX}

Mots-clés : industrie, industrie, histoire de l'industrie, histoire de l'industrie, innovation, innovation, marché, marché, technologie, technologie, produits, produits, importations, importations, exportations, exportations, Grèce, Grèce, époque contemporaine, époque contemporaine, histoire économique, histoire économique, histoire sociale, histoire sociale Keywords : industry, industrial history, innovation, market, technology, products, imports, exports, Greece, contemporary period, economic history, social history

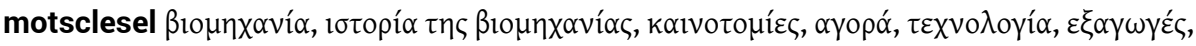

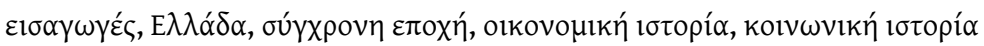

motsclestr endüstri, endüstri tarihi, yenilik, pazar, teknoloji, ürünler, ithalat, ihracat, Yunanistan, çağdaş dönem, sosyal tarik, ekonomik tarih

\section{AUTEUR}

\section{CHRISTINA AGRIANTONI}

Professeur émérite d'histoire, université de Thessalie, Grèce 